\title{
Deutsch-jüdische Periodika
}

\section{Rachel Heuberger / Hans Otto Horch / Gabriele von Glasenapp}

Seit ihrer Entstehung im 18. Jahrhundert war die deutsch-jüdische Presse ein wesentlicher Bestandteil der deutsch-jüdischen Publizistik und hat eine wichtige Rolle in der jüdischen Geschichte der Neuzeit gespielt. Ihre Bedeutung für die Erforschung des Judentums wird nun auch von der Wissenschaft in zunehmendem Maße wahrgenommen und im Hinblick auf die unterschiedlichen Funktionen thematisiert (Lappin und Nagel 2008, Vorwort). Zum einen stellt die deutsch-jüdische Presse eine unverzichtbare Ressource für die jüdische Geschichte dar und bietet, wie kaum ein anderes Medium, als Primärquelle unmittelbare Informationen $\mathrm{zu}$ allen Bereichen des jüdischen Lebens. In der Regel als aktuelle Nachrichtenblätter geschaffen, bilden die Periodika die unterschiedlichen religiösen, politischen, sozialen und kulturellen Richtungen innerhalb des Judentums ab. Zum anderen hat die deutsch-jüdische Presse aber auch als Forum der jüdischen Öffentlichkeit die historischen Ereignisse aktiv beeinflusst und als Agent gesellschaftlicher Diskurse die Auseinandersetzung um jüdische Identität mitbestimmt. In Anbetracht der nationalsozialistischen Zerstörungspolitik des jüdischen Kulturerbes besitzt sie zudem die Besonderheit eines authentischen Zeugnisses des einstmals blühenden jüdischen Lebens in seiner pluralistischen Ausprägung (Horch und Schicketanz 2001, 387).

Die bekanntermaßen problematische Begriffsbestimmung von ,jüdisch' trifft auch auf die Definition der deutsch-jüdischen Presse zu (Marten-Finnis und Winkler 2006, 9). In diesem Kontext werden nicht nur jene Druckerzeugnisse als Presse verstanden, die die allgemeinen Merkmale von Pressepublikationen wie Tages- und Wochenzeitungen besitzen, sondern auch Gemeindeblätter, Almanache, Kalender und Jahrbücher mit einbezogen. Zudem befassen sich entsprechende Publikationen inhaltlich maßgeblich mit jüdischen Themen, werden von jüdischen Herausgebern und Redakteuren getragen und für einen vorrangig jüdischen Leserkreis publiziert (Schwarz 2007, 46). Sprachlich umfasst die deutsch-jüdische Presse neben der Landessprache Deutsch auch Publikationen in Hebräisch, Jiddisch und Jüdisch-Deutsch, die an sich als per se ,jüdische' Sprachen gelten und in Deutschland nebeneinander bestanden haben. Geographisch gesehen gehören zur deutsch-jüdischen Presse nicht nur Periodika in den Grenzen des Kaiserreichs von 1871, sondern im gesamten deutschen Sprach- und Kulturraum.

Im Folgenden ist auf einige Periodika näher einzugehen, die für die Geschichte der deutsch-jüdischen Literatur durch Aufnahme von literarischen Werken und Literaturkritik besonders wichtig wurden (vgl. dazu Shedletzky 1986). Dabei kommt Kalendern, Almanachen und Jahrbüchern eine besondere Funktion zu, die in einem eigenen Abschnitt beleuchtet wird. 


\section{Die Entstehung der jüdischen Presse}

$\mathrm{Zu}$ den Vorläufern der jüdischen Presse zählen einzelne Zeitungen, die erstmals Ende des 17. Jahrhunderts für eine kurze Zeitspanne in Amsterdam erschienen. Als erste jüdische Zeitung überhaupt gilt die Gazeta de Amsterdam in judeo-spanischer Sprache, die sich 1675 an die dortige sephardische Gemeinde richtete (Encyclopaedia Judaica 2007, 486). Amsterdam, das Zentrum jüdischen Lebens im 17. und 18. Jahrhundert, war auch der Erscheinungsort der ersten Zeitung auf Jiddisch, der Kurantn, die 1686/1687 zweimal pro Woche in einer Dienstags- und FreitagsAusgabe erschien, sowie der ersten jüdischen Monatsschrift auf Hebräisch, Peri Ez Hayyim (1728-1761).

Erst seit der Mitte des 18. Jahrhunderts gibt es eine kontinuierliche jüdische Presse, die mit der Verbreitung der Ideen der Emanzipation auch die Gleichstellung der Juden und ihre Integration in die nichtjüdische Mehrheitsgesellschaft sowie eine geistige Erneuerung anstrebt. Die erste deutsch-jüdische Zeitschrift mit dem Titel Kohelet Musar, von Moses Mendelssohn begründet und erstmals 1750 als Wochenschrift erschienen, ist zugleich auch der Prototyp dieses neuen Mediums. Sie fungiert nicht nur als Agent der Informationsvermittlung, sondern soll dazu dienen, eine breite jüdische Öffentlichkeit richtungsweisend in das Gedankengut der Haskala und Emanzipation einzuführen. Gemäß ihrem Titel („Sammlung moralischer Lehren") legt die Zeitschrift den Schwerpunkt auf die Vermittlung ethischen Verhaltens und die Formierung eines bürgerlichen Wertekanons. Kohelet Musar war nach dem Vorbild der Moralischen Wochenschriften konzipiert, eines Typus von Zeitschriften, der in der ersten Hälfte des 18. Jahrhunderts geläufig war und wesentlich zur Verbreitung der Ideen der Aufklärung beigetragen hat. Der spezifisch jüdische Charakter der Zeitschrift liegt in der Bezugnahme auf die religiösen Werte des Judentums, wie sie in der klassischen jüdischen Literatur zum Ausdruck kommen, sowie in der hebräischen Sprache und Schrift, in der sie sich vorrangig an jüdische Jugendliche und Talmudschüler wendet, die sie mit den Gedanken der Haskala vertraut machen will (Nagel 2006, 27-42). Kohelet Musar stellte nach nur zwei Ausgaben ihr Erscheinen ein. Drei weitere Publikationen zählen zu den frühesten deutschjüdischen Presseerzeugnissen: die beiden 1752 von dem Arzt Benjamin Croneburg herausgegebenen Blätter Der kuriöse Antiquarius und Der große Schauplatz sowie die Dyhernfurter privilegirte Zeitung, die 1771/72 in Breslau erschien. Anders als Kohelet Musar sind die drei letztgenannten Publikationen zwar in hebräischer Schrift, jedoch in deutscher Sprache verfasst.

Im Gegensatz dazu entwickelt sich die Zeitschrift Ha-Meassef („Der Sammler“), von 1784 bis 1811 von Schülern Moses Mendelssohns in hebräischer Sprache herausgegeben, zum zentralen Organ der Aufklärer und ihrer Bestrebungen im deutschen und mitteleuropäischen Judentum. Der Gründer und langjährige Herausgeber des Ha-Meassef Isaac Euchel sowie seine Nachfolger gehören der zweiten Generation jüdischer Aufklärer an, die bereits mit den Ideen der Aufklärung vertraut sind 
und oftmals auch eine allgemeine Bildung außerhalb des traditionell jüdischen Rahmens erworben haben. Allerdings nimmt die Kenntnis des Hebräischen mit dem fortschreitenden Emanzipationsprozess und der Akkulturation der Juden ab, sodass die Zeitschrift im 19. Jahrhundert, als sich die überwiegende Mehrheit der deutschen Juden der deutschen Sprache zuwendet, an Bedeutung verliert. Zwar erscheinen in Deutschland auch weiterhin vereinzelt Titel in Hebräisch, bei diesen handelt es sich jedoch in der Regel um orthodoxe oder wissenschaftliche Veröffentlichungen. Erst mit dem Entstehen der zionistischen Bewegung in Deutschland zu Beginn des 20. Jahrhundert erscheinen erneut Zeitschriften auf Hebräisch. Die Kluft zwischen West- und Ostjudentum wird zudem dadurch verstärkt, dass die jiddische Sprache, die in Osteuropa eine bedeutende Rolle spielt, von den deutschen Juden nach Beginn des Emanzipationsprozesses nicht mehr verwendet wird. Die Zahl der jiddischsprachigen Zeitschriften bleibt sehr gering, und sie haben kaum einen Einfluss auf das kulturelle Leben der deutschen Juden.

Die Geschichte der jüdischen Presse in Deutschland bildet, wie Jacob Toury bereits in einer der ersten Studien zu diesem Thema $(1967,95)$ festgestellt hat, „bis in die dreißiger Jahre des 19. Jahrhunderts hinein eine Kette von ephemeren Versuchen, die kaum ein wirkliches Echo fanden.“ $\mathrm{Zu}$ den kurzlebigen Veröffentlichungen zählen die deutschsprachige, in hebräischen Lettern in Böhmen gedruckte „Jüdische Monatsschrift“ Jedidja, eine religiöse, pädagogisch ausgerichtete Halbjahresschrift (1817-1823), die Zeitschrift für die Reifere Jugend in Fürth (1817) sowie die Zeitschrift für die Wissenschaft des Judenthums (1822), die trotz ihrer Bedeutung (Mitarbeiter waren u.a. Leopold Zunz, Eduard Gans und Heinrich Heine) nur in einem Band erschien. Eine frühe Ausnahme stellt die nach der religiösen Reform ausgerichtete Sulamith dar, die von David Fraenkel und Joseph Wolf aus Dessau begründet wurde. Sie ist die erste jüdische Zeitschrift, die auf Hochdeutsch in Fraktur gedruckt wird, und erscheint kontinuierlich zwischen 1806 und 1848 in unregelmäßigen Abständen. Die Zeitschrift setzt sich für Reformen in der Liturgie ein, vor allem für die Abhaltung der Predigt in deutscher Sprache, von denen sie zahlreiche abdruckt, und propagiert ein weltoffenes und tolerantes Judentum, das in einer religiösen und kulturellen Erneuerung der Juden münden soll. Zur gleichen Zeit erscheint in Österreich von 1820 bis 1831 Bikkure ha-Ittim, ein Organ zur Verbreitung der Ideen der Aufklärung, das in den Jahren von 1825 bis 1831 die einzige regelmäßig publizierte jüdische Zeitschrift weltweit darstellt.

\section{Die deutsch-jüdische Presse im 19. Jahrhundert in ihren verschiedenen Strömungen}

Im politischen Vormärz entsteht eine Vielfalt von Zeitschriften, Zeitungen, Jahrbüchern und Kalendern, die der gesamten Bandbreite des jüdischen Lebens in seinen unterschiedlichen religiösen, politischen, sozialen und kulturellen Facetten Aus- 
druck verleihen. Mit der Ausbreitung der Reformbewegung unter den deutschen Juden dominiert bis zur Mitte des Jahrhunderts weiterhin die vorrangig reformorientierte Presse, die interessanten Lesestoff und zugleich praktische Unterweisung in religiösen Angelegenheiten bietet. Die Wochenschrift Der Israelit des 19. Jahrhunderts (1839-1848), von den Reformrabbinern Mendel Heß und Samuel Holdheim herausgegeben, enthält größtenteils Beiträge von Mendel Heß und fungiert, entsprechend der Einstellung der beiden Herausgeber, als Sprachrohr der radikalen Reformbewegung, die das Judentum in eine rein konfessionelle Religionsgemeinschaft ohne historisch-nationale Elemente verwandeln und den religiösen und bürgerlichen Fortschritt der Juden fördern will.

Die bedeutendste Zeitung ist jedoch zweifellos die Allgemeine Zeitung des Judenthums (AZJ, 1837-1922), die nicht nur durch ihren langen Erscheinungszeitraum unter den jüdischen Periodika deutscher Sprache eine besondere Stellung einnimmt (Horch 1985). Sie wurde 1837 von dem Rabbiner Ludwig Philippson begründet, der bis zu seinem Tod 1889 als Herausgeber fungierte, selbst zahlreiche Artikel veröffentlichte und für jedes Editorial verantwortlich zeichnete. Im Großformat der allgemeinen Presse erschien sie anfangs dreimal pro Woche, dann wöchentlich, zuletzt ab 1920 alle zwei Wochen, bis sie 1922 im Organ des Central-Vereins deutscher Staatsbürger jüdischen Glaubens (CV) aufging. Die AZJ deklariert sich im Untertitel als „ein unpartheiisches Organ für alles jüdische Interesse in Betreff von Politik, Religion, Literatur, Geschichte, Sprachkunde und Belletristik“ (AZJ 1837, 1) und hat sich zum Ziel gesetzt, den Zusammenhalt des deutschen Judentums zu stärken. Philippson achtete stets darauf, allen Strömungen des Judentums eine Plattform zu bieten, und räumte insbesondere der Kulturberichterstattung einen großen Raum ein. Gleichzeitig strebte er, dem gemäßigten Flügel der Reformbewegung angehörend, auch die Umsetzung innerjüdischer Reform innerhalb der Synagoge und der Erziehung an. Vorrangig blieb jedoch der Kampf um die vollständige Emanzipation der Juden in den deutschen Staaten und gegen den Antisemitismus in all seinen Formen sowie die Stärkung der jüdischen Solidarität, wie die ausführliche Berichterstattung der Zeitung über die sogenannte ,Damaskus-Affäre‘ im Jahr 1840 belegt, bei der Juden wahrheitswidrig des Ritualmords beschuldigt wurden.

Die $A Z J$ war durch ihre weite Verbreitung und Akzeptanz die einflussreichste deutsch-jüdische Zeitung, gewissermaßen eine Institution für sich, die im politischen Alltag der deutschen Juden durch ihr aktives Eintreten für gesellschaftliche Veränderungen und das Mitwirken bei der Gründung wichtiger Vereine und Institutionen (z.B. dem Leipziger „Institut zur Förderung der israelitischen Literatur“, einer Art jüdischer Buchgemeinschaft) eine große Rolle spielte. Artikel über allgemeine politische Ereignisse nehmen zu Beginn genauso viel Raum ein wie die Berichterstattung zu jüdischen Belangen. Bedeutende jüdische Persönlichkeiten, darunter Gabriel Rießer oder die Begründer der Wissenschaft des Judentums Leopold Zunz, Julius Fürst, Abraham Geiger und Samuel David Luzatto zählen zu den Auto- 
ren. Der Historiker Isaac Markus Jost widmete der Zeitung in seiner Geschichte der Israeliten ein eigenes Kapitel, in dem er deren Funktion als Plattform des innerjüdischen Diskurses betont und den Enthusiasmus von Philippson hervorhebt, dem es gelungen sei, seine Leser für jüdische Belange zu engagieren: „Sie [die $A Z J$ ] bildet [...] fortwährend einen Sammelplatz zur Erörterung sämmtlicher jüdischer Angelegenheiten, wie zugleich einen Unterhaltungssaal für diejenigen, welche neue Nachrichten und kleinere Geisteserzeugnisse und Blüthen aus dem jüdischen Leben kennenlernen wollen.“ (AZJ 11, 1847, 150). Nach der Reichsgründung 1870/71 rückte der Kampf gegen den zunehmenden Antisemitismus ins Zentrum. Unter Philippsons Nachfolger Gustav Karpeles richtete sich der Blick vermehrt nach Osten, während sich in der Ära Ludwig Geiger das Interesse auf eine antizionistische und antiorthodoxe Position verengte. In den letzten beiden Jahren von 1919 bis 1921 fungierte Albert Katz als Herausgeber.

Im Kontext der deutsch-jüdischen Literatur- und Kulturgeschichte kommt der AZJ eine besondere Bedeutung zu, weil sie von Beginn an die Produktion jüdischer wie nichtjüdischer Autoren als Seismograph der allgemeinen Entwicklung des deutsch- bzw. christlich-jüdischen Verhältnisses ansah und dementsprechend der Literaturkritik einen hohen Stellenwert einräumte: „Die Art, wie die Dichter einer Epoche die Juden schildern und Ansichten über sie aussprechen, giebt uns die in jener vorherrschenden Meinungen über sie besser und deutlicher kund, als selbst Gesetze und Thatsachen, welche aus besonderen Motiven hervorgegangen sein können.“ (AZJ 42, 1878, 787) Allerdings ändert sich im Verlauf ihres langen Erscheinens die Einstellung zu verschiedenen Autoren. Während Ludwig Philippson, geprägt durch den Idealismus der Klassik und Romantik, unter didaktischen Gesichtspunkten dem historisch-heroischen Genre der jüdischen Erzählliteratur einen Vorrang einräumte und selbst, ausgehend von dem bereits im ersten Jahrgang abgedruckten Roman seines Bruders Phöbus Philippson Die Marannen, als Autor entsprechend aktiv war (z.B. Sepphoris und Rom, 1866, Jakob Tirado, 1867), wurde Gustav Karpeles als ein Anhänger der realistischen Erzählliteratur Propagator des Genres der Dorf- und Ghettogeschichte, wobei er sich auf bekannte Autoren wie Berthold Auerbach, Leopold Kompert und vor allem Karl Emil Franzos berufen konnte. Die Suche Ludwig Geigers nach dem modernen jüdischen Roman seit der Jahrhundertwende blieb allerdings vergeblich - zu vielfältig war die Auseinandersetzung um jüdische Identität, zu disparat die Lösungsversuche im Rahmen der Moderne seit dem Naturalismus und ihrer Wiener Ausprägung, der ,Wiener Moderne‘.

Als Reaktion auf die Dominanz der Reformbewegung und der damit verbundenen zunehmenden Verbreitung der reformorientierten Zeitschriften begründete die Orthodoxie in Deutschland seit der Mitte des 19. Jahrhunderts ihre eigenen Periodika. Von 1845 bis 1854 erschien das „Organ zur Wahrung der Interessen des orthodoxen Judenthums“ Der Treue Zionswächter, herausgegeben von dem Altonaer Schuldirektor Samuel Enoch in Zusammenarbeit mit dem Oberrabbiner von Altona und 
Schleswig-Holstein Jakob Aaron Ettlinger. Die Zeitschrift kam zunächst wöchentlich, später dann in unregelmäßiger Abfolge heraus; ihre hebräische Beilage Shomer Tsion ha-Ne'eman (seit 1846) diente der Erörterung halachischer Fragestellungen und Diskussionen, ritueller Entscheidungen und der Verbreitung religiöser Lyrik. Erklärtes Ziel der Zeitschrift war es, Inhalte und Ausdrucksformen des orthodoxen Judentums als unverzichtbare und unveräußerliche Werte $\mathrm{zu}$ bewahren, daher wurden Reformen in Kultus und Ritus kategorisch abgelehnt und der Einfluss der Moderne auf das zeitgenössische Judentum als schädlich gebrandmarkt. Es gelang Ettlinger, der als einer der letzten großen Talmudgelehrten im deutschen Sprachraum gilt, mit dem Treuen Zionswächter für die Neo-Orthodoxie ein repräsentatives Forum zu schaffen und diese in der Öffentlichkeit als religiöse und auch politisch einflussreiche Partei nachhaltig zu positionieren (Meyer 1996, 164). Nach Einstellung der Zeitschrift folgten weitere orthodoxe Periodika, so in direkter Nachfolge Jeschurun und sechs Jahre später Der Israelit, beides Organe der neuerstarkten NeoOrthodoxie. Jeschurun. Ein Monatsblatt zur Förderung jüdischen Geistes und jüdischen Lebens, in Haus, Gemeinde und Schule (1854-1870) wird von Samson Raphael Hirsch herausgegeben, Rabbiner der Israelitischen Religionsgesellschaft zu Frankfurt am Main und Begründer der Neo-Orthodoxie und der Austrittsgemeinden in Deutschland. Jeschurun diente vorrangig als Plattform für Hirschs religiöse Positionierung, die er polemisch gegen seine religiösen Gegner Zacharias Frankel und Heinrich Graetz behauptete. Sie richtete sich ausschließlich an eine jüdische Leserschaft, die in ihrem Kampf gegen die Reformbewegung bestärkt werden sollte. Großen Einfluss auf die jüdische Öffentlichkeit hatte die im Jahr 1860 von Marcus Lehmann als „ein Zentralorgan für das orthodoxe Judentum“ begründete Zeitschrift Der Israelit, die einmal, ab 1884 zweimal pro Woche ohne Unterbrechungen erschien, bis sie im November 1938 von den Nationalsozialisten verboten wurde. Unter der Leitung ihres Gründers, des renommierten Rabbiners und Schriftstellers Marcus Lehmann, bildete die im Kampf gegen die Reformbewegung kompromisslose Zeitschrift das Gegengewicht zur liberal-reformerischen AZJ sowie deren Nachfolgerin, der CV-Zeitung. Der Israelit ist, vor allem nach der Übernahme des Jeschurun, über fast acht Jahrzehnte das bedeutendste Publikationsorgan der deutsch-jüdischen Orthodoxie und Sprachrohr der zu Beginn des 20. Jahrhunderts begründeten radikal antizionistischen Agudat Israel-Partei. Lehmann initiierte 1871 eine hebräische, 1873 eine jiddische Parallelausgabe des Blattes, das dadurch auch über die Grenzen des deutschsprachigen Raums hinaus wahrgenommen wurde. Nach seinem Tod 1890 übernahm sein Sohn Oscar Lehmann die Leitung der Zeitschrift. Auch in dieser Zeitschrift kam, wie in der AZJ, der Literaturkritik große Bedeutung zu, aber auch der Publikation von fiktionaler Literatur wie die historischen und gegenwartsorientierten Erzählungen und Romane des Herausgebers und der Tochter Samson Raphael Hirschs, Sara Hirsch, die bereits seit 1862 in Jeschurun Erzählungen mit eindeutig orthodoxer Ausrichtung veröffentlicht hatte. 
Von 1870 bis 1923 erschien die von Esriel Hildesheimer, dem Rabbiner und späteren Gründer des Rabbinerseminars in Berlin, initiierte wöchentliche Zeitschrift Die jüdische Presse, abwechselnd herausgegeben zuerst von Samuel Enoch, Gustav Karpeles und Jacob Hollander, in den Jahren von 1876 bis 1882 dann von Seligmann Meyer. Von 1883 bis zu seinem Tod 1910 fungierte Hirsch Hildesheimer als Herausgeber der Wochenschrift, die 1923 ihr Erscheinen einstellte. Gemäßigter in ihrem Umgang mit den Rabbinern der Reformbewegung, unterstützte die Zeitung die neue zionistische Bewegung und die Besiedlung Palästinas und entwickelte sich zu Beginn des 20. Jahrhunderts zum offiziellen Organ des ,Mizrachi', der religiösen Strömung innerhalb der zionistischen Bewegung (Bleich 1980). Von 1914 bis 1930 erschien eine neue Folge des Jeschurun, eine Monatsschrift für Lehre und Leben im Judentum. Herausgegeben von Joseph Wohlgemuth, bildete die Zeitschrift ein Sprachrohr des orthodoxen Berliner Rabbinerseminars und veröffentlichte vor allem die wissenschaftlichen Beiträge der Lehrer und Schüler des Seminars, in den Jahrgängen von 1920 bis 1926 auch in hebräischer Sprache.

\section{Wissenschaftliche Zeitschriften}

Das 19. Jahrhundert ist auch die Blütezeit der deutschsprachigen wissenschaftlichen Presse, die mit einer großen Anzahl einzelner, teils kurzfristig, teils über Jahrzehnte erscheinender Titel vor allem der Verbreitung der Wissenschaft des Judentums, der internen Kommunikation sowie ihrer öffentlichen Repräsentation diente. Bereits 1823 erschien unter der Redaktion von Leopold Zunz die Zeitschrift für die Wissenschaft des Judenthums als erstes wissenschaftliches Periodikum, das allerdings sein Erscheinen nach drei Heften eines Jahrgangs bereits wieder einstellen musste. Berücksichtigt man die Jahrbücher und Mitteilungsblätter wissenschaftlicher Institutionen und Vereine, so sind es über 30 verschiedene Titel, die im Laufe der Jahre erschienen, von denen hier nur die wichtigsten genannt werden können (Krone 2012). Zu den frühen Zeitschriften zählen die von Abraham Geiger von 1835 bis 1847 herausgegebene Wissenschaftliche Zeitschrift für Jüdische Theologie und Zacharias Frankels von 1844 bis 1846 veröffentlichte Zeitschrift für die religiösen Interessen des Judentums. Abraham Geiger, der bedeutendste Vertreter der jüdischen Reformbewegung, formuliert in seiner Zeitschrift die Grundsätze des reformorientierten Judentums und tritt für eine grundlegende Erneuerung des traditionellen jüdischen Ritus ein. In den Einzelbeiträgen werden zudem die zentralen Themen der jüdischen Literatur- und Kulturgeschichte abgehandelt. In unserem Zusammenhang wichtig wurde seine dort veröffentlichte Artikelserie über „,üdische Zeitschriften“ (1839-1844). Daran anknüpfend gab Geiger, der auch zu den bedeutendsten Wissenschaftlern seiner Zeit zählt, ab 1862 ein neues vierteljährlich erscheinendes Organ, die Jüdische Zeitschrift für Wissenschaft und Leben heraus. Auch diese Veröffentlichung war ein Versuch, die geistigen und sozialen Aufgaben des zeitgenössischen Judentums in 
der Epoche der Modernisierung neu zu definieren. Nach seinem Tod 1874 gab sein Sohn Ludwig Geiger 1875 noch einen Jahrgang heraus, dann stellte die Zeitschrift ihr Erscheinen ein. Ebenso wie ihr Herausgeber war die Zeitschrift von Seiten der Orthodoxie oftmals scharfer Kritik ausgesetzt.

Dagegen hatte die ebenfalls theologisch ausgerichtete, von Zacharias Frankel monatlich herausgegebene Zeitschrift für die religiösen Interessen des Judenthums nur eine kurze Lebensdauer (April 1844 bis Dezember 1846). Der Plan zur Fortsetzung als Vierteljahrsschrift wurde nicht realisiert. Zacharias Frankel, langjähriger Dresdner Oberrabbiner und Direktor des Breslauer Jüdisch-Theologischen Seminars, begründete mit seiner positiv-historischen, auf einen Ausgleich zwischen Reformern und Orthodoxen bedachten Theologie die gemäßigte Strömung des konservativen Judentums. Als Vorläuferin der von Frankel 1851 gegründeten Monatsschrift für Geschichte und Wissenschaft des Judentums widmet sich das Organ schwerpunktmäßig „der Geschichte und der innern Architectonik der jüdischen Theologie“, deren Bedingungen kritisch hinterfragt und auf der Basis eines modernen Wissenschaftsbegriffs teilweise revidiert wurden (Frankel, Prospectus, Dezember 1843, 6). Darüber hinaus verstand sich die Zeitschrift, an der zahlreiche namhafte Gelehrte der Zeit mitwirkten, als politisches Organ zur Abwehr der gegen das Judentum gerichteten Vorwürfe von außen.

Ebenfalls dem Fortschrittsgedanken der Emanzipation und der jüdischen Reformbewegung, sozusagen als „Instrument der Emanzipationsdebatte“ (Schapkow 2006, 505) sowie der Verbreitung der Wissenschaft des Judentums verpflichtet, verstand sich die von dem Orientalisten und Bibliographen Julius Fürst in Leipzig herausgegebene Zeitschrift Der Orient, Berichte, Studien und Kritiken für jüdische Geschichte und Literatur, die von 1840 bis 1851 jeweils wöchentlich mit einem Literaturblatt als ständige Beilage erschien. Der Orient setzte sich zum Ziel, seinen Lesern wissenschaftliche Beiträge zur jüdischen und der damit verwandten orientalischen Geschichte zu präsentieren, wobei die jüdische Literatur und Kultur als integraler Bestandteil der allgemeinen Kultur verstanden werden. Die Zeitung richtete sich an ein möglichst breites liberales, wissenschaftlich interessiertes Bildungsbürgertum und veröffentlichte in seinem Literaturblatt auch Beiträge christlicher Autoren. Mit seiner Fokussierung auf ein jüdisches Gesamtinteresse auf der Grundlage säkularer Bestimmung jüdischer Tradition und Geschichte wird Der Orient während der Dauer seines Erscheinens zu einer ernstzunehmenden Konkurrenz für die $A Z J$ und neben ihr zur erfolgreichsten deutsch-jüdischen Zeitschrift in den 1840er Jahren.

Die bedeutendste deutschsprachige wissenschaftliche Zeitschrift des 19. Jahrhunderts ist zweifelsohne die Monatsschrift für Geschichte und Wissenschaft des Judentums (MGWJ), „eines der imponierendsten Denkmäler, das sich der jüdische Geist in der Diasporageschichte geschaffen hat" (Wilhelm 1963, 327). Sie erschien, abgesehen von einer fünfjährigen Unterbrechung ab 1887, von 1851 bis 1939 zunächst monatlich, nach der Jahrhundertwende ca. alle zwei Monate, wobei der letz- 
te Band mit der Jahresangabe 1939 erst 1941 mit besonderer Druckerlaubnis als Jahrbuch erscheinen konnte, von der Gestapo jedoch beschlagnahmt und erst 1963 als Nachdruck in der ursprünglichen Fassung veröffentlicht wurde. Die $M G W J$ wurde von Zacharias Frankel begründet und entwickelte sich zum inoffiziellen Organ des von ihm geleiteten Jüdisch-Theologischen Seminars in Breslau sowie zum Veröffentlichungsforum der Ideen der von Frankel begründeten Richtung des konservativen Judentums. Sie entspricht in Aufbau und Inhalt den allgemeinen wissenschaftlichen Publikationen der Zeit - auf eine Anzahl wissenschaftlicher Aufsätze folgen Rezensionen und Mitteilungen - und erlangte durch ihr langes Erscheinen sowie die namhaften Herausgeber und Autoren ihr herausragendes Renommee. Nach Frankel, der hier seine Forschungen zur rabbinischen Literatur, insbesondere des Talmuds, publizierte, übernahm von 1869 bis 1897 mit Heinrich Graetz einer der namhaftesten jüdischen Historiker des 19. Jahrhunderts die Redaktion der Zeitschrift und verlagerte den Schwerpunkt fortan auf philologische und historische Studien. In den Jahren 1882 bis 1886 wurde er von Pinkus Friedrich Frankl, Abraham Geigers Nachfolger als Rabbiner in Berlin, als Mitherausgeber unterstützt. Nach fünfjähriger Pause übernahmen 1892 der Historiker Markus Brann und der Wissenschaftler David Kaufmann, beides Absolventen des Breslauer Seminars, die Herausgabe der Zeitschrift, die Brann nach Kaufmanns Tod von 1899 bis 1919 allein weiterführte. Seit 1920 fungierte der Philosophiehistoriker Isaak Heinemann als Herausgeber, die Veröffentlichung des letzten Bandes stellte der Rabbiner Leo Baeck, Präsident der Reichsvertretung der Deutschen Juden, zusammen. Bis heute dient die MGWJ, in deren Artikel alle Themenbereiche der Jüdischen Studien in objektiver Weise abgehandelt werden und rein theologische Auseinandersetzungen ausgeklammert bleiben, eine Zeitschrift, die über 500 der bedeutendsten Rabbiner und Gelehrten jener Zeit aus Deutschland und Mitteleuropa, aber auch aus den USA und Palästina zu ihren Beiträgern zählte, als Grundlage moderner Forschung.

Einige wissenschaftliche Zeitschriften beschränkten sich auf einen engen, einer Disziplin entsprechenden Themenbereich, so die historischen und bibliographischen Zeitschriften. Zur ersten Kategorie zählt als bekanntestes Organ die Zeitschrift für die Geschichte der Juden in Deutschland (ZGJD), die erste jüdische Zeitschrift, die sich ausschließlich der historischen Forschung widmete und in zwei Folgen (1887-1892, 1929-1937) erschien. Fachlich hoch angesehen, erreichte die Vierteljahresschrift der zweiten Folge, die von dem Historiker Ismar Elbogen, dem Bibliographen Aron Freimann und dem Nürnberger Rabbiner Max Freudenthal herausgegeben wurde, im Vergleich zur Monatsschrift nur ein geringes und sehr spezielles Fachpublikum. Zur zweiten Gruppe gehören die bibliographischen Publikationen, allen voran die in den Jahren 1858-1882 von Moritz Steinschneider herausgegebene Hebräische Bibliographie Ha-Mazkir sowie die von Heinrich Brody begründete und ab 1902 von Aron Freimann weitergeführte Zeitschrift für Hebräische Bibliographie (1896-1921). Beide sind hochspezialisierte Fachblätter, deren grundlegende wissenschaftliche Bedeutung ungeachtet des geringen Leserkreises unbestritten ist. 


\section{Politische und kulturelle Zeitschriften}

Gegen Ende des 19. Jahrhunderts entwickelte sich das neue Genre einer politisch ausgerichteten jüdischen Presse, die $\mathrm{zu}$ tagespolitischen und gesellschaftlichen Auseinandersetzungen in der deutschen Öffentlichkeit Stellung bezog und mit Beiträgen und Kommentaren in die Debatten eingriff. Der „Centralverein Deutscher Staatsbürger Jüdischen Glaubens“, 1893 als Reaktion auf den erstarkenden Antisemitismus gegründet und in der Weimarer Republik die einflussreichste jüdische Organisation in Deutschland, repräsentierte die Mehrheit der assimilierten bürgerlich konservativen oder liberalen Juden und beruhte auf der Überzeugung, dass die Juden eine ausschließlich religiöse Gemeinschaft seien, deren vollständige staatsbürgerliche Emanzipation sowie gleichberechtigte Teilhabe an der deutschen Kultur durchgesetzt werden müsse. Mit Im deutschen Reich (1895-1922) wurde ein Organ geschaffen, welches mit publizistischen Mitteln darauf abzielte, das Selbstbewusstsein der deutschen Juden in ihrem Kampf gegen Diskriminierung zu stärken und die Integration in den deutschen Kulturkreis durchzusetzen (Bitzer 2014). Gleichzeitig richtete sich die Zeitschrift in ihren Aufklärungsbemühungen gegen antijüdische Vorurteile auch an nichtjüdische Leser. Die Nachfolgezeitschrift, die C.V.-Zeitung (1922-1938), eine der bedeutendsten jüdischen Wochenzeitungen im deutschen Sprachraum, berichtete bis zu ihrem Verbot 1938 über alle Bereiche des jüdischen Lebens und intensivierte ihren Abwehrkampf gegen den militanter werdenden Antisemitismus in der Weimarer Republik, wobei sie immer um parteipolitische Neutralität bemüht war.

Ganz anders ist es bei den zionistisch orientierten Zeitschriften, die in dieser Zeit ebenfalls begründet wurden und die Ideen des Zionismus, der neuen jüdischen Nationalbewegung, offensiv vertraten. Die wichtigste zionistische Zeitschrift, die Jüdische Rundschau (1902-1938), die neben der C.V.- Zeitung zu den auflagenstärksten jüdischen Wochenzeitungen im deutschsprachigen Raum zählt, entwickelte sich zum politischen Gegner und zur Konkurrenz der C.V.-Zeitung im Kampf um die Zustimmung der deutschen Juden. Hervorgegangen aus dem Berliner Vereinsboten (1895-1901) und der Israelitischen Rundschau (1901-1902) ist die Jüdische Rundschau das offizielle „Organ der Zionistischen Vereinigung für Deutschland“ und zielt darauf ab, vor allem die Jugend für das zionistische Programm einer Heimstätte in Palästina zu gewinnen. Wechselnde Chefredakteure, darunter Julius Becker, Felix Abraham, Hugo Herrmann, Leo Herrmann, Fritz Löwenstein, Hans Klötzel, Robert Weltsch oder Hans Bloch, sind für die zunächst einmal wöchentlich, seit 1919 in der Regel in drei- bis viertägigem Abstand zweimal pro Woche erscheinende Zeitung verantwortlich. Die Jüdische Rundschau informierte in ihren Berichten über alle Bereiche jüdischen Lebens im In- und Ausland, seit 1932 erschienen auch zahlreiche Sonderhefte; nach 1933 rückten Informationen über Emigrationsmöglichkeiten für auswanderungswillige Leser ins Zentrum. Mit ihrer modernen, dezidiert kämpferi- 
schen Berichterstattung trug die Jüdische Rundschau wesentlich zur Politisierung der jüdischen Presse im deutschsprachigen Raum bei. Aufgrund ihres Engagements gegen den zunehmenden Antisemitismus steigerte die Zeitschrift ihre Auflage am Ende der Weimarer Republik auf 40.000 Exemplare. Nach dem Novemberpogrom 1938 musste die Jüdische Rundschau ihre Arbeit einstellen; ihre Nachfolgerin, die Jüdische Weltrundschau, erschien von 1939 bis 1949 zunächst in der Schweiz, später in Jerusalem.

Weitere zionistische Blätter des frühen 20. Jahrhunderts sind die kurzlebige Monatsschrift Altneuland (1904-1906), herausgegeben als „Organ der zionistischen Kommission zur Erforschung Palästinas“ von Franz Oppenheimer, Selig Eugen Soskin und Otto Warburg mit praxisorientierten Berichten zur Besiedlung des Landes Israel sowie das Zentralorgan der zionistischen Bewegung Die Welt (1897-1914), begründet von Theodor Herzl und herausgegeben von wechselnden Redakteuren.

Auf akademischer Ebene und in kleinem Kreis, aber auf einem sehr hohen intellektuellen Niveau wurde die ideologische Auseinandersetzung zwischen deutschem Nationalismus und Zionismus in den Blättern der jüdischen Studentenbewegung fortgesetzt. Die K.C.Blaetter (1910-1933), die Monatsschrift der im Kartell-Convent vereinigten Korporationen, vertrat eine deutsch-vaterländische Gesinnung und die Auffassung, die deutschen Juden seien durch Geschichte, Kultur- und Rechtsgemeinschaft integraler Bestandteil des deutschen Volkes. Alle zionistischen Bestrebungen wurden auf das entschiedenste abgelehnt. Demgegenüber war Der jüdische Student (1902-1933) als „Zeitschrift des Kartells Jüdischer Verbindungen“ das erste überregionale Verbandsorgan zionistischer Studentenvereine und vertrat uneingeschränkt das Programm der zionistischen Bewegung im Sinn einer Erneuerung des jüdischen Volkes und der Besiedlung des Landes Israel.

Zionistisch beeinflusst, aber vor allem auf die Herausbildung der kulturellen Renaissance der Juden ausgerichtet war die 1901 begründete Monatsschrift Ost und West (1901-1923), die in den ersten Jahren mit dem Untertitel „Illustrierte Monatsschrift für modernes Judentum“ erschien und deren erklärtes Ziel es war, den Assimilationsprozess der Juden in West- und Mitteleuropa durch die Konstruktion einer gemeinsamen ethnisch-nationalen jüdischen Identität unter Einbeziehung der osteuropäischen Ausprägung des Judentums aufzuhalten. Eine zentrale Rolle spielte die jüdische Kunst, durch deren Betonung der Wunsch der jüdischen Minderheit nach Beibehaltung ihrer ethnischen Unterschiedlichkeit geweckt und der Integrationswille in die deutsche Kultur geschwächt werden sollte (Brenner 1998, 52). Die Zeitschrift, deren Titel programmatisch für die Zusammenarbeit namhafter jüdischer Persönlichkeiten aus Ost- und Westeuropa stand und die zugleich eines der herausragenden Beispiele dieser Kooperation darstellte (Rosenfeld 1994, 83), widmete sich der Dokumentation künstlerischer, literarischer und wissenschaftlicher Leistungen mit besonderem Augenmerk auf die Kunst und betrieb aktiv die Vermittlung der Kulturleistungen der Ostjuden im Westen. Die Zeitschrift stand für die kul- 
turzionistische Position innerhalb der jüdischen Nationalbewegung, die mit der Entwicklung einer jüdischen Nationalkultur vorrangig die Schaffung eines geistigen Zentrums in Palästina und keine politische Lösung anstrebte. Martin Buber, der den Begriff der „Jüdischen Renaissance“ geprägt hat, suchte mit der von ihm herausgegebenen Monatsschrift Der Jude (1916-1928) die seit 1900 einsetzende Fraktionierung des Judentums im Sinn einer geistig-kulturellen Einigungsbewegung aufzuheben. Mit dem Titel bewusst auf die von Gabriel Rießer 1832 begründete kurzlebige Zeitschrift Der Jude. Periodische Blätter für Religion und Gewissensfreiheit (1832-1835) zurückgreifend, verschob Buber jedoch den Schwerpunkt von der bürgerlichen Gleichberechtigung des Einzelnen auf die Anerkennung des jüdischen Kollektivs: „Wir fordern nicht Gewissensfreiheit für die Angehörigen eines Glaubens, sondern Lebens- und Arbeitsfreiheit für eine niedergehaltene Volksgemeinschaft.“ (Der Jude 1, 1916/1917, 3). Unter besonderer Beachtung der von den assimilierten Westjuden oftmals missachteten ,Ostjuden“ und ihrer Kultur bot Der Jude ein Forum für die Diskussion literarischer, historischer, philosophischer, religiöser, politischer und soziologischer Fragen.

Eine kulturelle Zeitschrift, die von namhaften nationalliberalen Vertretern der jüdischen Wissenschaft, Politik und Kultur herausgegeben wurde, war die „Zeitschrift für Politik, Wirtschaft und Literatur in Ost und West“ Neue jüdische Monatshefte (1916-1920), die politisch neutral und als Forum für alle Strömungen des Judentums dem Ausgleich der jüdischen Parteien dienen sollte. Die Besonderheit der Zeitschrift besteht darin, dass sie sich explizit auch an das nichtjüdische Publikum richtete, um der Unkenntnis in jüdischen Dingen entgegenzuarbeiten mit dem Ziel, die Gleichberechtigung der Juden in Staat und Gesellschaft zu verwirklichen.

\section{Die Jüdische Presse in der Weimarer Republik}

In der Weimarer Republik machte sich die Renaissance jüdischen Lebens in der steigenden Anzahl von Zeitschriften jüdischer Vereine, Berufs- und Interessenverbände bemerkbar, für die exemplarisch hier nur die Titel Blätter des Jüdischen Frauenbundes, Jüdische Schulzeitung. Monatsschrift für Erziehung, Unterricht und Schulpolitik, das Organ des Reichsverbands der Jüdischen Lehrervereine, sowie Jüdische Arbeits- und Wanderfürsorge als eine der vielen Veröffentlichungen der jüdischen Wohlfahrtsverbände genannt werden können. Als neuer Typ jüdischer Zeitschriften reüssieren die Gemeindeblätter, unter denen das in Hamburg begründete Israelitische Familienblatt (1899-1938), das sich mit einer dezidiert politisch neutralen Haltung an die bürgerliche jüdische Öffentlichkeit wandte, am erfolgreichsten war. Gemeinsam mit der C.V.-Zeitung, der Jüdischen Rundschau und dem Gemeindeblatt Berlin zählt das Israelitische Familienblatt zu den auflagenstärksten Periodika in der Weimarer Republik und damit zu den „Großen Vier“ der jüdischen Presse jener Jahre (Strauss 1980, 326). Dagegen stand das Frankfurter Israelitische Familienblatt 
(1900-1923), das sich an die Mitglieder der Frankfurter Israelitischen Gemeinde richtete, aber auch überregional stark rezipiert wurde, der zionistisch-religiösen ,Misrachi'-Strömung nahe, die auf die Gründung einer jüdischen Heimstätte in Palästina auf der Grundlage der Torah und des jüdischen Religionsgesetzes abzielte.

Eine Vorreiterrolle in der Auseinandersetzung mit dem zunehmenden Antisemitismus nahm Der Schild (1922-1938) ein, das offizielle Organ des 1919 gegründeten ,Reichsbunds jüdischer Frontsoldaten', der sich die Abwehr des Antisemitismus explizit zur Aufgabe gemacht hatte und für die Assimilation der Juden in ihre deutsche Heimat eintrat. Bis zuletzt prägte die unterschiedliche Ausrichtung der deutsch-nationalen Orientierung einerseits und des Zionismus andererseits den Charakter der jüdischen Presse. Im Rahmen der PRESSA, der „Internationalen Presse-Ausstellung“, die 1928 in Köln gezeigt wurde, gab es eine erste Bilanz der jüdischen Presse, die sich unter dem Titel „Jüdische Sonderschau der PRESSA“ (JSOP) in einem eigenen Pavillon präsentierte (Marten-Finnis und Nagel, 2012). In Zeiten des wachsenden Antisemitismus wurde auch die deutsch-jüdische Presse zum ersten und bislang letzten Mal in ihrer Entwicklung und der Vielfalt ihrer publizistischen Aktivitäten in Hebräisch, Jiddisch und den Landessprachen im Detail dargestellt. Um der zunehmenden Diskriminierung entgegenzuwirken und die nichtjüdische Öffentlichkeit $\mathrm{zu}$ erreichen, gaben die beiden großen Zeitungen, die C.V.Zeitung seit 1925 und die Jüdische Rundschau, in den 1930er Jahren Monatsausgaben mit bereits veröffentlichten Artikeln heraus, in denen sie sich verstärkt der deutschen Politik zuwandten.

\section{Die Jüdische Presse im Dritten Reich}

Mit Beginn der nationalsozialistischen Herrschaft 1933 endete die Pressefreiheit; auch die jüdische Presse unterlag nun wachsenden Verboten und einer strengen Zensur. Bis zum 11. November 1938 erschienen jedoch noch knapp 150 jüdische Zeitungen und Zeitschriften in Deutschland, darunter auch die drei großen jüdischen Blätter Jüdische Rundschau, C.V.-Zeitung und Israelitisches Familienblatt. Einige teilweise kostenlose Gemeinde- und Vereinsblätter wurden neu gegründet, so etwa die „Monatsblätter des Kulturbunds deutscher Juden“ Jüdischer Kulturbund, der von 1933 bis 1938 in Berlin erschien, sodass von einer Scheinrenaissance gesprochen werden konnte (Freeden 1987). Frei von nationalsozialistischer Propaganda fungierten die jüdischen Publikationen fortan als Organe der jüdischen Rückbesinnung und bewegten sich durch die Stärkung der eigenen Kultur und der Selbstbehauptung zwischen Anpassung und Widerstand. Unter den Bedingungen der Diskriminierung und Ausgrenzung bewahrte die jüdische Presse in Deutschland die kritische Distanz journalistischer Professionalität in der Beurteilung der Geschehnisse und diente gleichzeitig als Organ der jüdischen Gemeinschaft nach außen. 
Nach dem Verbot der jüdischen Presse in Folge der Pogromnacht vom 9. und 10. November 1938 wurde das von den Nationalsozialisten initiierte und kontrollierte Jüdische Nachrichtenblatt begründet, das vom 22. November 1938 bis zum 4. Juni 1943 in Berlin erschien (Maier, 2008). Das Organ wurde vom Propagandaministerium gesteuert und diente vor allem der Bekanntmachung der antijüdischen Verordnungen und Gesetze für die Juden in Deutschland sowie als Mitteilungsblatt zu Fragen und gezielten Förderung der Emigration. Chefredakteur war bis zu seinem Tod im November 1942 Leon Kreindler, Mitglied des Vorstandes der Jüdischen Gemeinde Berlin und ehemaliger Schriftleiter des Berliner Gemeindeblattes sowie der Berliner Ausgabe des Israelitischen Familienblatts. Das Jüdische Nachrichtenblatt war vor allem ein Instrument der Verfolgungspolitik, das den jüdischen Journalisten in ihrer Arbeit eine äußerst schwierige Gratwanderung in der Beachtung der Auflagen der Zensur abverlangte.

\section{Die Jüdische Presse in der Gegenwart}

Die ersten Periodika nach 1945 setzten sich aus einer Vielzahl von Lagerzeitungen zusammen, die direkt nach der Befreiung in den sogenannten jüdischen ,Displaced Persons Camps ‘ (DP-Lagern) veröffentlicht wurden, in denen sich die Überlebenden der Judenvernichtung unter der Besatzung der Alliierten in Deutschland sammelten. Eine beeindruckende Zahl von über 100 regionalen und überregionalen Blättern und Zeitschriften wurde bis zur Auflösung der Lager in den 1950er Jahren veröffentlicht, die überwiegende Anzahl in jiddischer Sprache, so auch die erste, im Oktober 1945 in lateinischer Schrift gedruckte Jiddischer Landsberger Cajtung im bayrischen DP-Lager Landsberg.

Die Jüdische Allgemeine, 1946 in Düsseldorf als regionales Gemeindeblatt für die Nord-Rheinprovinz und Westfalen gegründet und von 1973 bis 2002 unter dem Titel Allgemeine Jüdische Wochenzeitung, seit 2002 unter dem Titel Jüdische Allgemeine veröffentlicht, spielte für den Neubeginn der jüdischen Presse und das jüdische Leben in Deutschland nach dem Krieg eine zentrale Rolle. Herausgegeben vom Zentralrat der Juden in Deutschland erscheint die Zeitung seit 1999 einmal wöchentlich in Berlin und ist das auflagenstärkste Periodikum des deutschen Judentums, das sich als „Wochenzeitung für Politik, Kultur, Religion und jüdisches Leben“ nach Eigendefinition als Nachfolge in der publizistischen Tradition der großen jüdischen liberalen Blätter des 19. Jahrhunderts sieht. Seit 2002 erscheint in Berlin ein neues überregionales Monatsblatt in Russisch, die Jewreskaja Gaseta, die einen explizit kritischen Standpunkt gegenüber dem Zentralrat und seiner Politik einnimmt und versucht, sich als Sprachrohr der liberalen jüdischen Gemeinden zu positionieren. Die Zeitung wendet sich an die rund 90.000 russisch-jüdischen Zuwanderer, die die Mehrheit innerhalb der jüdischen Gemeinden bilden, daneben veröffentlichen die Herausgeber der Zeitung, die Werner Media Group, zwei weitere Zeitungen auf Rus- 
sisch. Seit 2005 erscheint auch eine identische Ausgabe in deutscher Sprache mit dem Namen Jüdische Zeitung. Des Weiteren erscheinen in unregelmäßigen Abständen eine Anzahl von Gemeinde- und Mitteilungsblättern jüdischer Organisationen.

$\mathrm{Zu}$ den frühen wissenschaftlichen Zeitschriften der Nachkriegszeit zählen das seit 1956 im Ausland verantwortete LBI Year Book des Leo Baeck Instituts London und die Frankfurter Judaistischen Beiträge, die vom Seminar für Judaistik der Frankfurter Universität seit 1973 in unregelmäßigen Abständen herausgegeben werden. Seit den 1980er Jahren folgten weitere größere und kleinere Zeitschriften, darunter 1991 Aschkenas, 1996 das vom Fritz Bauer Institut veröffentlichte Jahrbuch zur Geschichte und Wirkung des Holocaust und seit 1998 die vom Salomon Ludwig Steinheim-Institut vierteljährlich veröffentlichte Zeitschrift Kalonymos. Beiträge zur deutsch-jüdischen Geschichte.

An ein breites nichtjüdisches Publikum wandte sich auch die 1962 begründete Vierteljahresschrift „Zeitschrift zum Verständnis des Judentums“ Tribüne, die als Abwehrreaktion gegen vorhandene antisemitische Tendenzen entstand. Die Vierteljahresschrift, die ein vom Rechtsextremismus über jüdische Geschichte und Shoah bis zu religiösem Fanatismus und Nahostkonflikt reichendes Themenspektrum umfasst, musste nach 51 Jahren Ende 2012 mit Heft 204 ihr Erscheinen einstellen und bietet seither eine Homepage an, auf der weiterhin Beiträge und Interviews zu aktuellen Themen veröffentlicht werden.

Zahlreiche Publikationen erscheinen mittlerweile gleichzeitig oder auch ausschließlich als elektronische Ausgabe im Internet, so z.B. HaGalil online, eine Internet-Seite, die sich nach eigenen Angaben als das „größte jüdische online-Magazin in deutscher Sprache“ definiert. Die Auswirkungen der digitalen Transformation auf die Entwicklung der deutsch-jüdischen Presse in der Zukunft sind gegenwärtig ebenso schwer einzuschätzen wie die Prognosen für die Entwicklung einer pluralistischen und facettenreichen jüdischen Gemeinschaft in Deutschland, die in welcher Form auch immer (online/digital oder traditionell) zum Entstehen einer divergierenden deutsch-jüdischen Presse führen wird.

Rachel Heuberger, Hans Otto Horch

\section{Jüdische Kalender, Almanache und Jahrbücher}

Anders als im Fall der jüdische Presse existieren zu den deutsch-jüdischen Almanachen, Kalendern und Jahrbüchern bislang nur marginale Forschungsansätze, sodass der aktuelle Kenntnisstand bezüglich der Geschichte dieser Periodika bis heute gravierende Lücken aufweist. Zur Kenntnis genommen wurde diese Ausprägung periodisch erscheinender Literatur bislang weder durch Forschungen über die jüdische Presse noch durch die umfangreiche (nichtjüdische) Almanach-Forschung. Daher mangelt es nach wie vor an verlässlichen bibliographischen Nachweisen des 
Produktionsumfanges, d.h. der derzeitige Wissenstand basiert auf den von Aron Freimann (1905) und William Popper (1905) zu Beginn des 20. Jahrhunderts erstellten Verzeichnissen, die das erste Drittel des 20. Jahrhunderts nicht mehr erfassen konnten. Eine weitere Aufstellung unternahm Johannes Valentin Schwarz (2002) ein Jahrhundert später, er konzentrierte sich jedoch ausschließlich auf die erste Hälfte des 19. Jahrhunderts. Hinzu kommt ein gutes Dutzend von Aufsätzen, die sich jedoch lediglich einzelnen Vertretern dieses Mediums widmeten, sowie Recherchen von Gabriele von Glasenapp (2006), sodass das Feld der deutschsprachigen jüdischen Kalender - ein Begriff, der im Folgenden als Sammelbezeichnung für die Kalender, Almanache, Jahr- und Taschenbücher in ihrer Gesamtheit verwendet werden soll - bis heute wissenschaftlich eine Terra incognita darstellt.

\section{Die Anfänge}

Jüdische Kalender sind ein transnationales Phänomen. Fast in jedem Land mit jüdischer Gemeinschaft hat es jüdische Kalender gegeben; allerdings ist die Massierung dieses Mediums in den deutschsprachigen Ländern Mitteleuropas besonders stark für die gut 120 Jahre zwischen dem Erscheinen des ersten deutschsprachigen jüdischen Almanachs im Jahr 1818 und dem endgültigen Verbot der jüdischen Presse im Jahr 1938 - lassen sich zwischen 45 und 50 in Inhalt, Aufmachung, Ausrichtung und Erscheinungsdauer höchst unterschiedliche Kalender nachweisen.

Die Geschichte der jüdischen Kalender geht jedoch weiter zurück als in das 19. Jahrhundert, denn bereits für das 16. Jahrhundert lässt sich der Druck von zahlreichen Kalendern, damals noch ,Luach‘ genannt, nachweisen (Cassuto 1932, 810-813). Diese ,Luchot' bestanden zunächst ausschließlich aus kalendarischen Tabellen, in denen Fest- und Fasttage sowie Sabbatperikopen, meist gleich für mehrere Jahre, verzeichnet waren. Ende des 16. Jahrhunderts gab es jedoch bereits ,Luchot“, die auch für ein einzelnes (jüdisches) Jahr jeweils neu gedruckt wurden. Neben der Angabe sämtlicher Tage des betreffenden Jahres enthielten sie nun auch Angaben über den christlichen Kalender und christliche Feste sowie Verzeichnisse über lokale Märkte und Messen. Bei diesen ,Luchot' handelt es sich um reine Gebrauchspublikationen, die im Unterschied zu den im letzten Drittel des 18. Jahrhunderts aufkommenden nichtjüdischen Almanachen (Bunzel 1999) keinerlei literarischen, wissenschaftlichen, politischen oder unterhaltenden Charakter aufweisen. Analog zu den nichtjüdischen Kalendern stammen jedoch auch die Leser dieser ,Luchot eher aus den sozialen Unterschichten (Knopf 1999, 122), etwa Händler oder Hausierer, die nach deren Angaben ihre Routen festlegen konnten, wussten, in welcher Stadt wann Markt abgehalten wurde, wann sie wegen christlicher Feiertage keinen Handel treiben durften und wann die eigenen Feiertage begannen. 


\section{Die Einflüsse der Haskala}

Die Geschichte der deutschsprachigen jüdischen Kalender im engeren Sinn, d.h. jener Veröffentlichungen, die periodisch einmal im Jahr erschienen (meist Ende des alten oder kurz nach Beginn des neuen jüdischen Jahres), im Titel entweder die Bezeichnung Kalender, Almanach oder Jahrbuch trugen, mitunter ein Kalendarium aufwiesen, deren Hauptteil jedoch aus literarischen, wissenschaftlichen, politischen, künstlerischen, pädagogischen oder weltanschaulichen Beiträgen verschiedener Autoren bestanden, ist - wie die Geschichte der deutschsprachigen jüdischen Presse - eng verbunden mit der Geschichte der Haskala, d.h. dem Beginn der jüdischen Akkulturations- und innerjüdischen Reformbewegung. Die erste Generation von Herausgebern, Redakteuren und Mitarbeitern jüdischer Kalender waren daher fast ausnahmslos engagierte Vertreter der Haskala.

So standen bei der Gründung des ersten jüdischen Kalenders 1818, des Almanach für die israelitische Jugend, durch den Berliner Pädagogen Jeremias Heinemann vor allem die pädagogischen Aspekte des Kalenders im Vordergrund. Jugendlichen Lesern jüdischer Herkunft sollte auf diese Weise eine Alternative zur nichtjüdischen und nicht selten explizit christlich orientierten Jugendliteratur geboten werden.

Dem Geist der Aufklärung und der Emanzipationsbewegung des beginnenden 19. Jahrhunderts verpflichtet, hatte Heinemann in den Almanach Texte von jüdischen, aber auch nichtjüdischen Autoren aufgenommen. Als Verfasser der einzelnen Beiträge, die mehrheitlich aus Parabeln, Gedichten, Epigrammen und Rätseln bestanden, finden sich daher neben jüdischen Autoren, Rabbinern und Pädagogen - unter ihnen Maimon Fränkel, Lippmann Büschenthal, Leopold Zunz, David Friedländer, Karl Siegfried Günsburg und Gotthold Salomon - auch christliche Autoren. Die von Heinemann intendierte Verbindung zwischen jüdischer und nichtjüdischer Literatur zeigt sich auch im Titelkupfer des Almanachs, auf dem der mittig gesetzte Titel mit der expliziten Adressierung „für die israelitische Jugend“ und der Nennung des jüdischen Jahres umrahmt wird von Vignetten, die die vier Jahreszeiten abbilden, d.h. keinerlei jüdische Bezüge aufweisen.

Heinemanns Unternehmen kann nicht nur als typisch für die späte Haskala angesehen werden, sondern auch als typisch für die jüdischen Kalender in ihrer Gesamtheit. Bereits nach drei Jahrgängen wurde der Almanach ohne Angaben von Gründen wieder eingestellt; es bleibt, wie auch bei späteren Unternehmungen, unklar, ob eine zu geringe Leserschaft, mangelnde finanzielle Mittel oder die zahlreichen anderen Verpflichtungen des Herausgebers die Verantwortung für die Kurzlebigkeit dieses ersten jüdisch-literarischen Kalenders tragen. 


\section{Kalender und Almanache des 19. Jahrhunderts}

Der tatsächliche Durchbruch des Genres erfolgte erst gut zwei Jahrzehnte später, denn zu Beginn der 1840er Jahre wurden unabhängig voneinander gleich mehrere jüdische Kalender ins Leben gerufen, die in Ausstattung, Ausrichtung, Inhalt, Titel und ihrer sehr unterschiedlichen Erscheinungsdauer bereits fast die gesamte Bandbreite des jüdisch-literarischen Kalenderwesens im 19. Jahrhundert repräsentieren.

$\mathrm{Zu}$ diesen Kalendern zählen der von dem Prediger und Lehrer Selig Louis Liepmannssohn edierte Gemeinnützige Volkskalender für Israeliten (1841), der 1840 einmalig erscheinende Israelitische Musen-Almanach des Hechinger Rabbiners und Publizisten Samuel Mayer, dessen erklärte Intention es war, das ehemalige und gegenwärtige jüdische „Nationalleben durch Erzählungen, Novellen und Gedichte“ der Vergessenheit zu entreißen (Mayer 1840, V), sowie das von Carl Maien und Sigmund Frankenberg edierte Taschenbuch Jeschurun. Taschenbuch für Schilderungen und Anklänge aus dem Leben der Israeliten auf das Jahr 5601.

Ein gemeinsames Kennzeichen dieser drei Veröffentlichungen besteht darin, dass ihnen eher ein Almanach-Charakter immanent war, d.h. es dominierten literarisch-pädagogische Beiträge, ein Kalendarium fehlt bezeichnenderweise ganz. Zudem blieben trotz positiver Resonanz in der jüdischen Presse alle drei Kalender einmalige Unternehmungen. Gleichzeitig erschienen zu Beginn der 1840er Jahre jüdische Kalender auf dem Markt, deren Inhalte bereits auf eine ganz andere Auffassung dieses Mediums verweisen. Erstmals wurde hier nämlich der Versuch unternommen, in Aufmachung und Inhalten an die Form des alten ,Luach“ anzuknüpfen: so enthielten diese Kalender am Anfang ein ausführliches Kalendarium, an das sich in einem zweiten Teil die Beiträge, meist unter der Bezeichnung ,Jahrbuch“ zusammengefasst, anschlossen. Die Tatsache, dass bei diesen Beiträgen mitunter ein anderer Druckort angegeben war als bei dem kalendarischen Teil, lässt vermuten, dass beide Teile sowohl gemeinsam als auch getrennt verkauft wurden, wobei das Kalendarium als Gebrauchsartikel für jüdische Kaufleute und Händler höchstwahrscheinlich einen deutlich höheren Absatz erzielte und dazu diente, mit seinem Erlös wenigstens in Teilen das ,Jahrbuch“ mitzufinanzieren.

Gleichzeitig aber sollten diese Kalender der 1840er Jahre auch das inhaltliche Spektrum erheblich erweitern und modernisieren. Das gilt in erster Linie sowohl für den von dem Lehrer Karl Klein herausgegebenen Volkskalender für Israeliten. Mit einem Jahrbuch des Nützlichen und Unterhaltenden, der seit 1841 zunächst im schlesischen Brieg, später in Breslau erschien, als auch für das von dem Publizisten Isidor Busch ab 1842 in Wien veröffentlichte Periodikum Kalender und Jahrbuch, der erste österreichische Kalender in deutscher Sprache. Bereits in der Einladung zur Subskription auf Kleins Kalender, die in verschiedenen jüdischen Zeitschriften abgedruckt wurde, kündigte der Herausgeber das breite inhaltliche Spektrum seines Kalenders an: 
1) den gewöhnlichen astronomischen und chronologischen Kalender. 2) den jüdischen Kalender mit deutschen und hebräischen Lettern. 3) die Veränderungen des Mondes nach seinem verschiedenen Eintritt in den Meridian. 4) Sonnen- und Mondes- Auf- und Untergang, Tageslänge. 5) Witterungskalender. 6) Zeitrechnung, Festrechnung. Gang- und Sichtbarkeit der Planeten. Sonnen- und Mond-Finsternisse. 7) Tafel zur Stellung der Uhr. 8) Jahrmarktsverzeichniß. 9) Genealogie der hohen Häuser. 10) Verzeichniß der Postcurse. So viel als Kalender; als Jahrbuch aber: 1) kurzer Abriß der jüdischen Geschichte von Moses Mendelssohn bis auf die neueste Zeit. 2) Biographien berühmter Israeliten. Anekdoten und Charakterzüge aus dem Leben edler Israeliten. 3) das jüdische Kalenderwesen. 4) Erklärung der jüdischen Feste. 5) Parabeln. Legenden. Miscellen. 6) Sammlung der die religiöse und bürgerliche Verfassung der Israeliten in den preußischen Staaten betreffenden Gesetze, Verordnungen u.s.w. 7) Wechselrecht. Kaufmännisches Recht. (Klein 1841, 228)

Klein und Busch gewannen eine Vielzahl von Mitarbeitern für ihr Unternehmen, deren Namen sie gleichsam als Werbemaßnahme auf das Titelblatt des jeweiligen Jahrgangs drucken ließen. Zu diesen Mitarbeitern zählen Rabbiner und Prediger (ausschließlich der reformorientierten Richtung) wie Abraham Kohn, Salomon Formstecher, Eduard Kley und Joseph Aub, Literaten wie Isaac Assur Francolm, Raphael Fürstenthal und David Honigmann, Wissenschaftler, darunter Isaak Markus Jost, Eduard Munk, Abraham Tendlau und Meyer Isler, Pädagogen, darunter Leopold Stein, Jakob Auerbach und Raphael Kirchheim. Ihre Beiträge über religiöse, wissenschaftliche, politische, literarische und pädagogische Themen, die in den 1850er Jahren noch um eine nach Sparten aufgegliederte literarische Rundschau ergänzt wurden, prägten die Kalender von Klein und Busch in maßgeblicher Weise. Auffällig war zudem die deutliche Politisierung beider Kalender; abgedruckt wurden u.a. Gesetze und Verordnungen, die die religiöse und bürgerliche Verfassung der Israeliten in den preußischen und österreichischen Staaten betreffen und die mit vorsichtiger Hoffnung kommentiert werden (vgl. u.a. Honigmann 1847, 85-123).

Zur gleichen Zeit wurden Kalender bereits auch zum Gegenstand des innerjüdischen Literaturdiskurses. In seinem Überblick über die Entwicklung jüdischer Presseerzeugnisse zu Beginn der 1840er Jahre weist Abraham Geiger vor allem der periodischen Literatur eine entscheidende Rolle innerhalb der innerjüdischen Reformbewegungen zu. Ein wichtiger Bestandteil, oder wie Geiger es formuliert, „ein gesunder Zweig an dem Baume der periodischen Litteratur" (Geiger 1844, 390) bildeten die jüdischen Kalender. In seinen Ausführungen entwirft Geiger zugleich die Kriterien, die für die Beurteilung dieses Mediums anzulegen seien: „Ein jüdisches Jahrbuch muß einen religiösen, historischen und gemeinnützigen Gehalt haben, der allerdings nicht blos in fasslicher Rede, sondern auch mit dichterischem Schmucke auftreten mag; das eigenthümlich ästhetische Element darf auch eine Stelle einnehmen, aber nicht ausschließlich verwalten.“ (Geiger 1844, 388) Gefordert wird weiterhin ein korrektes Kalendarium, den neuesten Erkenntnissen der Wissenschaft verpflichtende Zeittafeln, die als Erinnerungsmedium bedeutender Ereignisse der jüdischen Geschichte dienen sollen, sowie unterhaltende, d.h. belletristische Teile 
jüdischen Inhalts. Es „ziemt solchen Jahrbüchern“ aber auch, so fordert Geiger weiter, „das wahrhaft religiöse Element und der Nachweis, wie sich dasselbe in jüdischem Leben und jüdischer Sitte abspiegelt“ (Geiger 1844, 390), und nicht zuletzt „eine Übersicht des vergangenen Jahres, die aber den Zeitraum des jüdischen Jahres umfassen sollte“ (Geiger 1844, 389).

Geigers Forderungen markieren den hohen Stellenwert, der Kalendern mittlerweile beigemessen wurde. Ein Abbild jüdischen Lebens sollten sie sein - und dies auf wissenschaftlicher, literarischer, pädagogischer und nicht zuletzt auch auf religiöser Ebene. Für die Zeitgenossen stellten die Kalender von Klein und Busch, auf die sich die Darlegungen von Geiger offensichtlich bezogen, Musterbeispiele dieses Mediums dar, denn die Kalendergründungen der folgenden Jahrzehnte übernahmen, mit leichten Modifikationen und Akzentverschiebungen, sowohl das kalendarische wie inhaltliche Schema dieser beiden herausragenden Vorgänger.

\section{Die Prager Kalender}

Das gilt vor allem für die böhmischen und hier an erster Stelle für die Prager Kalender. Vorreiter bildete hier der erstmals 1852 erscheinende Illustrirte israelitische Volkskalender. Als Herausgeber und Redakteur zeichnete der Prager Verleger Wolf Pascheles verantwortlich (Glasenapp 2011, 121-124). Verglichen mit den Kalendern von Busch und Klein war hier der literarisch-unterhaltende Akzent viel stärker ausgeprägt. Deutlich zeigen sich hier auch die Gegebenheiten des literarischen Marktes in Prag. So finden sich unter den Mitarbeitern des Kalenders zahlreiche Autoren, die bereits mit anderen Publikationen im Verlag von Pascheles an die Öffentlichkeit getreten waren, sodass der Verleger seinem Publikum ein sich ständig wiederholendes Gefühl des Wiedererkennens bereits bekannter Namen bieten konnte - ein nicht unwichtiger Faktor für den erfolgreichen Absatz des Kalenders. Pascheles ging jedoch noch einen Schritt weiter, indem er seinen Kalender offensiv als Werbeträger für die Produkte seines Verlages nutzte. Dieser Werbeteil umfasste zunächst nur die Einbandrückseite sowie die Rückseiten des Kalenders, wurde jedoch im Verlauf der folgenden Jahre und Jahrzehnte kontinuierlich erweitert, bis er im 20. Jahrhundert mehr als ein Drittel des gesamten Kalenders ausmachte.

Ganz offensichtlich war der Prager bzw. böhmische Markt mit dem Kalender von Wolf Pascheles, der nach seinem Tod von dessen Sohn Jakob weitergeführt wurde, keineswegs gesättigt, denn 1874 begann Jakobs Bruder Samuel mit der Edition eines eigenen Kalenders, der unter demselben Titel erschien wie der seines Bruders. Mitte der 1890er Jahre zog sich Jakob Pascheles aus dem Verlagsgeschäft zurück, und der von ihm edierte Illustrierte Israelitische Volkskalender wurde ohne Angaben von Gründen eingestellt. Der von Samuel Pascheles bzw. ab 1909 von seinem Sohn Alfred edierte Kalender existierte hingegen noch bis 1939, d.h. bis zur Besetzung und Zerschlagung der Tschechoslowakei durch die deutsche Wehrmacht. 
Ein Indiz für die ungebrochene Beliebtheit jüdischer Kalender gerade in Prag bildet die Tatsache, dass Anfang der 1880er Jahre der Buchhändler und Verleger Jakob W. Brandeis, ein Schwiegersohn von Wolf Pascheles und damit Schwager der Brüder Jakob und Samuel, ebenfalls auf die Idee kam, einen Kalender auf den Markt zu bringen. Sowohl in der Wahl des Titels als auch auf inhaltlicher Ebene kopierte Brandeis die Vorbilder der Brüder Pascheles; so existierten für insgesamt fünfzehn Jahre in Prag drei Kalender mit gleichem Titel - Illustrierter Israelitischer Volkskalender -, sodass man gezwungen war, zur Unterscheidung den Namen des jeweiligen Herausgebers voranzusetzen. Brandeis' Kalender erschien in insgesamt 52 Jahrgängen, bis er 1932 nach dem Tod von Richard Brandeis, dem Sohn des Begründers, eingestellt wurde.

\section{Neo-orthodoxe Kalender}

Im Unterschied zu den jüdischen Zeitschriften, die immer auch ein Schauplatz innerjüdischer Auseinandersetzungen gewesen waren, gab es sowohl in der ersten Generation jüdischer Kalender, die von ausgewiesenen Anhängern der Reformbewegung ediert wurden, als auch in den in der zweiten Hälfte des 19. Jahrhunderts erschienenen Kalendern, deren Herausgeber mehrheitlich als gemäßigt konservativ charakterisiert werden können, kaum Angriffe gegen andere innerjüdische Richtungen; es lässt sich eindeutig das Bestreben erkennen, auf diese Weise einen möglichst großen Leserkreis aus allen innerjüdischen Strömungen zu gewinnen.

Wahrscheinlich aus diesem Grund erschien der erste explizit orthodoxe Kalender auch erst Anfang der 1880er Jahre. Bereits sein Titel, Volks-Kalender des Israelit, verweist auf seine Herkunft; er wurde auf Initiative von Marcus Lehmann ins Leben gerufen, dem Herausgeber der in Mainz erscheinenden orthodoxen Zeitschrift Der Israelit. Bezeichnenderweise unterscheidet sich dieser orthodox ausgerichtete Kalender kaum von seinen nicht-orthodoxen Vorgängern, er war vorwiegend literarisch ausgerichtet, politische, pädagogische und wissenschaftliche Beiträge waren selten, und die einzige Konzession an die orthodoxe Leserschaft bildete ein kontinuierlich erweitertes Verzeichnis von Gaststätten und Hotels, in denen jüdische Reisende den rituellen Vorschriften entsprechend essen und nächtigen konnten.

\section{Zionistische Kalender}

Dagegen führte das Aufkommen zionistisch orientierter Kalender gegen Ende des 19. Jahrhunderts zu einer weiteren Hochblüte des jüdischen Kalenderwesens. Analog zur vergleichsweise moderaten Haltung anderer jüdischer Kalender verstanden sich auch die zionistischen Organe weniger als Plattform zur Verbreitung zionisti- 
schen Gedankenguts denn als ein Forum für die in Deutschland noch wenig verbreitete osteuropäische jüdische Literatur, der hier sowohl durch den Abdruck von Übersetzungen als auch durch entsprechende literaturkritische Aufsätze Vorschub geleistet wurde (Glasenapp 2001, 55-57). So machte Theodor Zlocisti in seiner Funktion als Redakteur des von 1897 bis 1900 in Köln erscheinenden Jüdischen Volkskalenders zwar keinen Hehl aus der dezidiert zionistischen Ausrichtung seines Kalenders, angestrebt war jedoch in erster Linie die Schaffung eines jüdischen Volksbuches zur Vermittlung osteuropäischer jüdischer Literatur. In Deutschland war diesem Unterfangen zunächst nur wenig Erfolg beschieden, anders sah es mit den Kalendern in der Habsburgermonarchie aus, die mit ähnlichen Konzepten an die Öffentlichkeit traten. Das gilt vor allem für den seit 1902 im Brünner Verlag von Max Hickl erscheinenden Jüdischen Volkskalender, der damit warb, der ,einzige zionistische Kalender in deutscher Sprache“ zu sein, und zudem in einer sehr hohen Auflage von über 10.000 Stück vertrieben wurde. Die lange Laufzeit - auch Hickls Kalender wurde erst nach 37 Jahren, d.h. nach der deutschen Besetzung der Tschechoslowakei, eingestellt - spricht zugleich für die hohe Akzeptanz, die dieser Kalender offensichtlich weit über die Grenzen Böhmens hinaus hatte. Das gilt, wenngleich in geringerem Maße, auch für den seit 1915 in Wien erscheinenden von Otto Abeles und Ludwig Bato redigierten Jüdischen Nationalkalender, der sich allerdings auf Dauer offensichtlich nicht gegen den Konkurrenten aus Brünn behaupten konnte und Anfang der 1920er Jahre nach nur sechs Jahrgängen wieder eingestellt wurde.

Flankiert wurden diese im weitesten Sinne (kultur-)zionistisch ausgerichteten Kalender von zahlreichen Einzelunternehmungen, von denen sicherlich die bekannteste der 1902 von Berthold Feiwel edierte Jüdische Almanach (vgl. Bertz 1996) darstellt. Er enthielt anders als die zahlreichen hier angeführten Kalender kein Kalendarium mehr, sondern ausschließlich literarische Beiträge. Feiwels Intention, durch diesen Almanach „östliches und westliches Judentum in einem kleinen Abbild ihres Schaffens vorzuführen“ (Feiwel 1902, 14), markierte den entscheidenden Durchbruch der jüdisch-osteuropäischen Literatur in Deutschland. Als Indiz für den Erfolg des Almanachs können die kurz darauf erscheinende zweite Auflage gelten, das große und ausschließlich positive Echo in der jüdischen wie nichtjüdischen Presse sowie die zahlreichen Versuche jüdischer Publizisten in den Jahren bis 1939, das Konzept von Feiwels Almanach zu kopieren.

Dazu zählen der von der ,Vereinigung jüdischer Hochschüler aus Galizien BarKochba‘ 1910 in Wien herausgegebene Jüdische Almanach, der von Raphael Chamizer 1921 in Leipzig edierte Jüdische Almanach, der von Ludwig Bato 1925 ebenfalls in Wien redigierte Jüdische Almanach sowie der von Friedrich Thieberger und Felix Weltsch zwischen 1925 und 1938 in Prag zunächst im Auftrag des ,Keren Kayemeth“, danach im Auftrag der Zeitschrift Die Selbstwehr edierte Jüdische Almanach, der einzige dieser Almanache, der in mehreren Jahrgängen erschien (Kyselá 2003). Die unmittelbare Rezeption von Feiwels Almanach erstreckt sich jedoch auch auf die 
nicht deutschsprachigen Nachbarländer. So erschien bereits 1911 in Budapest der erste Jahrgang des von Simon Hevesi edierten Cultur-Almanachs in ungarischer Sprache, und 1922 gab Markus Krämer in Czernowitz den Juedischen Almanach für Gross-Rumänien heraus, dessen Beiträge in deutscher und rumänischer Sprache verfasst waren.

Fast ein Alleinstellungsmerkmal der zionistischen Kalender ist das ihnen durchweg sehr umfangreich beigegebene Bildmaterial. Vor allem die Bilder von Ephraim Moses Lilien und Hermann Struck wurden in diesem Kontext immer wieder abgedruckt, verliehen den Kalendern durch ihre Neuartigkeit und Ausdrucksstärke zusätzlich eine große Anziehungskraft und setzten zugleich künstlerische Standards, die auch die Kalender der 1920er und 1930er Jahre in entscheidender Weise prägen sollten.

Einer der bedeutendsten Kalender dieser Epoche war der von dem Berliner Rabbiner Emil Bernhard Cohn von 1928 bis 1936 redigierte Jüdische Kinderkalender (Völpel 1997, Richter 2002, Glasenapp 2012, 637-639). Obwohl dieser Kalender wie die meisten seiner Vorgänger ein jüdisches wie nichtjüdisches Kalendarium enthielt, trug er aufgrund seiner inhaltlichen Vielfalt eher den Charakter einer Anthologie als eines Kalenders. So enthielt er eine Vielfalt an literarischen Gattungen, aber auch Bastelanleitungen, Preisrätsel, Biographien sowie Berichte aus der jüdischen Jugendbewegung und aus Palästina. Seinen innovativen Charakter demonstrierte der Kalender in der Vielzahl an Illustrationen, die in ihrem Stil an aktuelle Entwicklungen der zeitgenössischen deutschen wie russischen Kunst anknüpften, in dem Aufruf an die Leser, sich an Text und Illustrationen mit eigenen Beiträgen zu beteiligen, hierfür wurde auf analoge Praktiken in anderen jüdischen (Jugend-) Zeitschriften zurückgegriffen, sowie in seiner Mischung von historischen Beiträgen und Themen mit eindeutigem Aktualitätsbezug. So wurden die Leser vor 1933 beispielsweise explizit dazu ermutigt, sich gegen antisemitische Angriffe offensiv zur Wehr zu setzen. Nach der Machtübergabe an die Nationalsozialisten ist hingegen eine deutliche Zunahme der Berichte über Palästina als möglichen zukünftigen Lebensraum des deutschen Judentums zu verzeichnen. Die Ausnahmeposition dieses Kalenders innerhalb der jüdischen Kinder- und Jugendliteratur der Weimarer Republik wird nicht zuletzt dadurch unter Beweis gestellt, dass er von Beginn an, trotz seiner zunächst eindeutig reformorientierten Positionen, uneingeschränkte Zustimmung auch von zionistischer wie auch von neo-orthodoxer Seite erhielt.

Zusammenfassend lässt sich festhalten, dass jüdische Kalender, Almanache, Jahrund Taschenbücher seit dem Beginn des 19. Jahrhunderts ein ebenso wichtiges wie heterogenes Forum innerjüdischer religiöser, pädagogischer, wissenschaftlicher, kulturpolitischer und literarischer Diskussionen bildeten, an denen sich bedeutende Rabbiner, Prediger, Literaten, Pädagogen und Wissenschaftler der Zeit intensiv beteiligten. In ihrer Bedeutung können Kalender durchaus der deutschsprachigen 
jüdischen Presse an die Seite gestellt werden, eine Tatsache, die auch den komplexen Wechselwirkungen zwischen diesen unterschiedlichen Periodika geschuldet ist. So wurden die Diskurse über die Inhalte der Kalender ausschließlich in der jüdischen Presse geführt und zwar mehrheitlich im Rahmen von Rezensionen der jährlich neu erscheinenden Kalender. In Zeitschriften wurden Kalender aber nicht nur rezensiert, sie druckten auch die Ankündigungen $\mathrm{zu}$ deren Subskription ab und warben für neu erscheinende Kalender. Hinzu kamen nicht selten enge personelle Verflechtungen zwischen den Herausgebern und Redakteuren von Kalendern und Zeitschriften.

Die große Anzahl der Kalender, ihre Vielfalt, die sich bereits im 19. Jahrhundert immer weiter ausdifferenzierte, belegt anschaulich, dass ihr Einfluss auf das jüdische Selbstverständnis und ihre Bedeutung für die innerjüdische Selbstvergewisserung nicht unterschätzt werden darf. Darüber hinaus können sie als reichhaltige, bislang allerdings noch nicht hinreichend erforschte Materialsammlungen zur jüdischen Pressegeschichte des 19. und 20. Jahrhunderts gelten.

Gabriele von Glasenapp

\section{Literaturverzeichnis}

Bertz, Inka: „Jüdischer Almanach 5663“. Hessing, Jakob (Hrsg.): Jüdischer Almanach 1996/5756 des Leo Baeck Instituts. Frankfurt a.M.: Jüdischer Verlag im Suhrkamp Verlag, 1995, 10-24.

Bitzer, Dominic: Im deutschen Reich. Das publizistische Organ des Centralvereins deutscher Staatsbürger jüdischen Glaubens. Aachen, Univ.-Diss., 2013. Online-Publikation über die Hochschulbibliothek der RWTH.

Bleich, Judith: „The Emergence of an Orthodox Press in Nineteenth-Century Germany“. Jewish Social Studies 42 (1980), 323-344.

Brenner, David A.: Marketing Identities. The Invention of Jewish Ethnicity in ,Ost und West'. Detroit: Wayne State University Press, 1998.

Bunzel, Wolfgang: „Almanache und Taschenbücher“. Fischer, Ernst; Haefs, Wilhelm; Mix, YorkGothart (Hrsg.): Von Almanach bis Zeitung. Ein Handbuch der Medien in Deutschland 1700-1800. München: C.H. Beck, 1999, 24-35.

C[assuto], U[mberto]. „Kalender“. Encyclopaedia Judaica. Berlin: Eschkol, 1932, Bd. 9, 810-813.

Diehl, Katrin: Die jüdische Presse im Dritten Reich. Zwischen Selbstbehauptung und Fremdbestimmung. Tübingen: Niemeyer, 1997.

Eikenberg, Gabriel: Der Mythos deutscher Kultur im Spiegel jüdischer Presse in Deutschland und Österreich von 1918 bis 1938. Hildesheim, Zürich, New York: Olms, 2010.

Feiwel, Berthold: „Geleitwort“. Feiwel, Berthold (Red.): Jüdischer Almanach 5663. Berlin: Jüdischer Verlag [1902], 9-16.

Fraenkel, Josef u.a. „Press“. Berenbaum, Michael; Skolnik, Fred (Hrsg.): Encyclopaedia Judaica. Detroit: Macmillan Reference USA, ${ }^{2} 2007$, Bd. 16, 486-505.

Frankel, Zacharias: „Prospectus“. Zeitschrift für die religiösen Interessen des Judenthums 1 (1843), 1-6. Online verfügbar über die Universitätsbibliothek Frankfurt a.M.

Freeden, Herbert A.: Die jüdische Presse im Dritten Reich. Frankfurt a.M.: Jüdischer Verlag bei Athenäum, 1987. 
Freimann, Aron: „Almanac“. Jewish Encyclopaedia. New York: Funk und Wagnalls, 1905, Bd. 1, 416-428.

Geiger, Abraham: „Jüdische Zeitschriften“. Wissenschaftliche Zeitschrift für Jüdische Theologie 4 (1839), 286-292, 459-471; 5 (1844), 372-390, 446-477.

Glasenapp, Gabriele von: „,Eine neue und neuartige Epoche‘. Ostjüdische Literatur in deutschjüdischen Zeitschriften und Almanachen vor dem Ersten Weltkrieg“. Krobb, Florian; StrümperKrobb, Sabine (Hrsg.): Literaturvermittlung um 1900. Fallstudien zu Wegen in das deutschsprachige kulturelle System. Amsterdam, New York: Rodopi, 2001, 45-60.

Glasenapp, Gabriele von: „Jüdische Kalender, Almanache und Jahrbücher. Zur Geschichte und Bedeutung eines ,vergessenen“ Mediums“. Marten-Finnis, Susanne; Winkler, Markus (Hrsg.): Die jüdische Presse im europäischen Kontext 1686-1990. Bremen: edition lumière, 2006, 73-88.

Glasenapp, Gabriele von: „,Die Meisterwerke der Poesie und Wissenschaft den Massen zugänglich machen'. Brandeis' Jüdische Universal-Bibliothek. Geschichte, Programm und Profil einer Prager Verlagsreihe“. Leipziger Jahrbuch zur Buchgeschichte 19 (2011), 117-171.

Glasenapp, Gabriele von: „Jüdische Kinder- und Jugendliteratur“. Hopster, Norbert (Hrsg.): Die Kinder- und Jugendliteratur der Weimarer Republik. Frankfurt a.M. u.a.: Peter Lang, 2012, 609-647.

H[erlit]z, G[eorg], und M[endel], P[robst]. „Presse jüdische“. Herlitz, Georg; Kirschner, Bruno (Hrsg.): Jüdisches Lexikon. Ein enzyklopädisches Handbuch des jüdischen Wissens in vier Bänden. Berlin: Jüdischer Verlag, 1927-1930, Bd. 4 (1930), Sp. 1102-1110.

Hirsch, Samson Raphael: „Prospectus“. Jeschurun 1 (1854), 1-4. Online verfügbar über die Universitätsbibliothek Frankfurt a.M.

Honigmann, David: „Die Judenfrage auf dem ersten vereinigten Landtag Preußens“. Volks-Kalender für Israeliten auf das Jahr 5608. Breslau: Joh. Urban Kern, 1847, 85-123.

Horch, Hans Otto: Auf der Suche nach der jüdischen Erzählliteratur. Die Literaturkritik der „Allgemeinen Zeitung des Judentums“ (1837-1922). Frankfurt a.M. u.a.: Peter Lang, 1985.

Horch, Hans Otto; Schicketanz, Till: „Ein getreues Abbild des jüdischen Lebens. Compact Memory Ein DFG-Projekt zur retrospektiven Digitalisierung jüdischer Periodika im deutschsprachigen Raum“. Menora. Jahrbuch für deutsch-jüdische Geschichte 12 (2001), 387-405.

Jost, Isaak Markus: Geschichte der Israeliten seit der Zeit der Makkabäer bis auf unsere Tage. Berlin: Schlesinger 1847, Bd. 10. Online verfügbar über die Universitätsbibliothek Frankfurt a.M.

Jüdische Presse im 19. Jahrhundert. Katalog zur Ausstellung in Tel Aviv 1967. Aachen: Internationales Zeitungsmuseum, 1967.

[Klein, Karl]: „,[Anzeige für Kleins ,Volkskalender für Israeliten‘]“. Der Orient. Berichte, Studien und Kritiken für jüdische Geschichte und Literatur 2 (1841), 228.

Knopf, Jan: „Kalender“. Fischer, Ernst; Haefs, Wilhelm; Mix, York-Gothart (Hrsg.): Von Almanach bis Zeitung. Ein Handbuch der Medien in Deutschland 1700-1800. München: C.H. Beck, 1999, 120-136.

Krone, Kerstin von der: Wissenschaft in Öffentlichkeit. Die Wissenschaft des Judentums und ihre Zeitschriften. Berlin: De Gruyter, 2012.

Kysela, Miroslava: „Die Protagonisten des Jüdischen Almanachs“. Balser, Bernd; Świattowska, Irena (Hrsg.): Annäherungen. Polnische, deutsche und internationale Germanistik. Wroctaw: Ofizyna Wydawnicza ATUT, 2003, 231-239.

Lappin, Eleonore: Der Jude 1916-1928. Jüdische Moderne zwischen Universalismus und Partikularismus. Tübingen: Mohr (Siebeck), 2000.

Lappin, Eleonore; Nagel, Michael (Hrsg.): Frauen und Frauenbilder in der europäsch-jüdischen Presse von der Aufklärung bis 1945. Bremen: edition lumière, 2007. 
Lappin, Eleonore; Nagel, Michael (Hrsg.): Deutsch-jüdische Presse und jüdische Geschichte. Bd. 2, Bremen: edition lumière, 2008.

Maier, Clemens: „Das jüdische Nachrichtenblatt 1938-1943. Instrument der Verfolgung und Mittel der Selbstbehauptung“. Marten-Finnis, Susanne; Winkler, Markus (Hrsg.): Deutsch-jüdische Presse und jüdische Geschichte. Dokumente, Darstellungen, Wechselbeziehungen. Bremen: edition lumière, 2008, Bd. 2, 164-178.

Marten-Finnis, Susanne; Winkler, Markus (Hrsg.): Die jüdische Presse im europäischen Kontext 1686-1990. Bremen: edition lumière, 2006.

Marten-Finnis, Susanne; Winkler, Markus: „Zur Einführung“. Marten-Finnis, Susanne; Winkler, Markus (Hrsg.): Die jüdische Presse im europäischen Kontext 1686-1990. Bremen: edition lumière, 2006, 9-16.

Marten-Finnis, Susanne; Winkler, Markus (Hrsg.): Deutsch-jüdische Presse und jüdische Geschichte. Dokumente, Darstellungen, Wechselbeziehungen. Bremen: edition lumière, 2008.

[Mayer, Samuel]: „Vorwort. Am Vortage des Neujahrsfestes 5600“. Mayer, Samuel (Hrsg.): Israelitischer Musen-Almanach. Dinkelsbühl 1840, V-VIII.

Meyer, Michael A.: „Jüdisches Selbstverständnis“. Meyer, Michael A. (Hrsg.): Deutsch-jüdische Geschichte der Neuzeit. München: C.H. Beck, 1996, Bd. II, 135-176.

Nagel, Michael: „Zur Journalistik der frühen Haskala“. Marten-Finnis, Susanne; Winkler, Markus (Hrsg.): Die jüdische Presse im europäischen Kontext 1686-1990. Bremen: edition lumière, 2006, 27-42.

Philippson, Johanna: „Ludwig Philippson und die Allgemeine Zeitung des Judentums“. Liebeschütz, Hans; Paucker, Arnold (Hrsg.): Das Judentum in der Deutschen Umwelt 1800-1850. Studien zur Frühgeschichte der Emanzipation. Tübingen: Mohr (Siebeck), 1977, 243-291.

Popper, William: „Periodicals“. Jewish Encyclopaedia. New York: Funk und Wagnalls, 1905, Bd. 9 , 616-639.

Marten-Finnis, Susanne; Nagel, Michael (Hrsg.): Die PRESSA. Internationale Presseausstellung Köln 1928 und der jüdische Beitrag zum modernen Journalismus. 2 Bde. Bremen: edition lumière, 2012.

Richter, Dieter: „Der jüdische Kinderkalender. 1928-1936. Ein Beitrag zur Geschichte der deutschjüdischen Kinderliteratur“. Nagel, Michael (Hrsg.): Zwischen Selbstbehauptung und Verfolgung. Deutsch-jüdische Zeitungen und Zeitschriften von der Aufklärung bis zum Nationalsozialismus. Hildesheim: Olms, 2002, 281-294.

Rosenfeld, Gavriel D.: „Defining ,Jewish Art“ in Ost und West, 1901-1908. A Study in the Nationalisation of Jewish Culture“. Leo Baeck Institute Yearbook 39 (1994), 83-110.

Schapkow, Carsten: „Die Zeitschrift Der Orient. Publizistik für die ,jüdischen Gesamtinteressen “ im Zeitalter der Emanzipation “. Wendehorst, Stephan (Hrsg.): Bausteine einer jüdischen Geschichte der Universität Leipzig. Leipzig: Universitätsverlag, 2006, 505-517.

Schwarz, Johannes Valentin: „,Ew. Exzellenz wage ich unterthänig vorzulegen.' Zur Konzessionierung und Zensur deutsch-jüdischer Periodika in den Königreichen Preußen und Sachsen bis 1850“. Nagel, Michael (Hrsg.): Zwischen Selbstbehauptung und Verfolgung. Deutsch-jüdische Zeitungen und Zeitschriften von der Aufklärung bis zum Nationalsozialismus. Hildesheim: Olms, 2002, 101-138.

Schwarz, Johannes Valentin: „,A New German-Jewish Public Sphere‘. Konzeptionelle Überlegungen zu einer Gesamtgeschichte der jüdischen Presse in Deutschland von der Aufklärung bis zur Gegenwart“. Marten-Finnis, Susanne; Bauer, Markus (Hrsg.): Die jüdische Presse. Forschungsmethoden - Erfahrungen - Ergebnisse. Bremen: edition lumière, 2007, 39-72.

Schwarz, Johannes Valentin: „,Zur Wahrung der Würde des Judentums‘. Jüdische Sonderschau (JSOP) auf der Kölner PRESSA im Spannungsfeld der religiösen Weltanschauungsgruppen“. Marten-Finnis, Susanne; Nagel, Michael (Hrsg.): Die PRESSA. Internationale Presseausstellung 
Köln 1928 und der jüdische Beitrag zum modernen Journalismus. 2 Bde. Bremen: edition lumière, 2012, Bd. 1, 229-280.

Shedletzky, Itta: Literaturdiskussion und Belletristik in den juedischen Zeitschriften in Deutschland 1837-1918. Jerusalem, Hebrew Univ. Diss., 1986 [Masch.].

Strauss, Herbert A.: „The Jewish Press in Germany, 1918-1939 (1943)“. Flinker, David; Rosenfeld, Shalom; Tsanin, Mordechai (Hrsg.): The Jewish Press that was. Accounts, Evaluations and Memories of Jewish Papers in pre-Holocaust Europe. Jerusalem: Jerusalem Post Press, 1989, 321-354.

Toury, Jacob: Die jüdische Presse im österreichischen Kaiserreich. Ein Beitrag zur Problematik der Akkulturation 1802-1918. Tübingen: Mohr (Siebeck), 1983.

Toury, Jakob: „Die Anfänge des jüdischen Zeitungswesens in Deutschland“. Bulletin des Leo Baeck Instituts 38-39 (1967), 93-123.

Völpel, Annegret: „1928 - The first issue of The Jewish Children’s Calendar, edited by Emil Bernhard Cohn, is published [...]".Gilman, Sander L.; Zipes, Jack (Hrsg.): Yale Companion to Jewish Writing and Thought in German Culture 1096-1996. New Haven, London: Yale University Press, 1997, 485-491.

Wilhelm, Kurt: „Die Monatsschrift für Geschichte und Wissenschaft des Judentums, ein geistesgeschichtlicher Versuch“. Kisch, Guido (Hrsg.): Das Breslauer Seminar. Jüdisch-Theologisches Seminar (Fraenckelscher Stiftung) in Breslau 1854-1938. Gedächtnisschrift. Tübingen: Mohr (Siebeck), 1963, 327-349. 